Pacific

Journal of

Mathematics

A REMARK ON INSTABILITY OF HARMONIC MAPS BETWEEN SPHERES

TÔRU NAKAJIMA 


\title{
A REMARK ON INSTABILITY OF HARMONIC MAPS BETWEEN SPHERES
}

\author{
TÔRU NAKAJIMA
}

We give a new characterization of the identity map between spheres.

\section{Introduction}

Let $k$ be an integer greater that two, and $\mathbb{S}^{k}$ be a $k$-dimensional unit Euclidean sphere. For a smooth map $u: \mathbb{S}^{k} \rightarrow \mathbb{S}^{k}$, the energy density $e(u)$ is the function $e(u): \mathbb{S}^{k} \rightarrow \mathbb{R}$ defined by $e(u)(x)=|d u(x)|^{2}\left(x \in \mathbb{S}^{k}\right)$, where $|d u(x)|$ is the Hilbert-Schmidt norm of a linear map $d u(x) \in T_{x}^{*} \mathbb{S}^{k} \otimes T_{u(x)} \mathbb{S}^{k}$. The Dirichlet energy $\mathbf{E}(u)$ of a smooth map $u: \mathbb{S}^{k} \rightarrow \mathbb{S}^{k}$ is defined by

$$
\mathbf{E}(u)=\frac{1}{2} \int_{\mathbb{S}^{k}} e(u) d \mu,
$$

where $\mu$ is the canonical measure induced by the Riemannian metric of $\mathbb{S}^{k}$. We often regard $u$ as a map from $\mathbb{S}^{k}$ to $(k+1)$-dimensional Euclidean space $\mathbb{R}^{k+1}$ and denote

$$
u(x)=\left(u_{1}(x), \ldots, u_{k+1}(x)\right) .
$$

A map $u$ is called a harmonic map if it is a critical point of the functional $\mathbf{E}$. They necessarily satisfy the Euler-Lagrange equation

$$
\Delta_{\mathbb{S}^{k}} u+|d u|^{2} u=0 \text { in } \mathbb{S}^{k},
$$

where $\Delta_{\mathbb{S}^{k}}$ is the Laplacian on $\mathbb{S}^{k}$. All harmonic maps are assumed to be smooth in this paper.

Let $u^{-1} T \mathbb{S}^{k}$ be the pull-back bundle of $T \mathbb{S}^{k}$ by $u$ and $C^{\infty}\left(u^{-1} T \mathbb{S}^{k}\right)$ be the vector space of smooth sections of $u^{-1} T \mathbb{S}^{k}$. For $\phi \in C^{\infty}\left(u^{-1} T \mathbb{S}^{k}\right)$, we consider a oneparameter variation

$$
u_{t} \in C^{\infty}\left((-\epsilon, \epsilon), C^{\infty}\left(\mathbb{S}^{k}, \mathbb{S}^{k}\right)\right), \quad \epsilon>0,
$$

MSC2000: primary 53C43, 58E20; secondary 35J45, 35J50.

Keywords: harmonic maps, instability, spheres.

This work was partially supported by Grant-in-Aid for Young Scientists (B), No. 18740088. 
such that $u_{0}=u$ and

$$
\left.\frac{\partial u_{t}}{\partial t}(x)\right|_{t=0}=\phi(x)
$$

The second variation $\delta_{u}^{2} \mathbf{E}$ of $\mathbf{E}$ at $u$ is a functional on $C^{\infty}\left(u^{-1} T \mathbb{S}^{k}\right)$ defined by

$$
\delta^{2} \mathbf{E}_{u}(\phi)=\left.\frac{d^{2}}{d t^{2}} \mathbf{E}\left(u_{t}\right)\right|_{t=0}
$$

and has an expression

$$
\delta_{u}^{2} \mathbf{E}(\phi)=\int_{\mathbb{S}^{k}}\left\{\left|\nabla^{\prime} \phi\right|^{2}-\langle\operatorname{Tr} R(\phi, d u) d u, \phi\rangle\right\} d \mu .
$$

where $\nabla^{\prime}$ is the induced connection on $C^{\infty}\left(u^{-1} T \mathbb{S}^{k}\right), R$ is a Riemannian curvature of $\mathbb{S}^{k}$ and $\operatorname{Tr}$ is a trace. We define the number $\lambda_{1}(u)$ by

$$
\lambda_{1}(u)=\inf _{\substack{\phi \in C^{\infty}\left(u^{-1} T \mathbb{S}^{k}\right) \\ \phi \neq 0}} \frac{\delta_{u}^{2} \mathbf{E}(\phi)}{\|\phi\|_{L^{2}\left(\mathbb{S}^{k}\right)}^{2}}
$$

where

$$
\|\phi\|_{L^{2}\left(\mathbb{S}^{k}\right)}=\left(\int_{\mathbb{S}^{k}}|\phi|^{2} d \mu\right)^{1 / 2} .
$$

By the result of Xin [1980], if $u$ is not a constant harmonic map, then $\lambda_{1}(u) \leq 2-k$. (On instability of harmonic maps, see also [Eells and Lemaire 1983], [Urakawa 1993] and [Xin 1996].) The purpose of this paper is to prove the following.

Theorem 1.1. Suppose $k \geq 3$ and $u: \mathbb{S}^{k} \rightarrow \mathbb{S}^{k}$ is a harmonic map. If $\lambda_{1}(u)=2-k$, then there exists $a(k+1) \times(k+1)$ orthogonal matrix $R$ satisfying

$$
u(x)=R x, \quad x \in \mathbb{S}^{k} .
$$

Remark. For any nonconstant harmonic map $u: \mathbb{S}^{2} \rightarrow \mathbb{S}^{2}$, the number $\lambda_{1}(u)$ equals 0 , since they minimize the Dirichlet energy in their homotopy classes and the Dirichlet energy is conformal invariant in this case.

\section{Proof of Theorem 1.1}

We need the following lemma from [Ramanathan 1986]. (See also [Nakajima 2003].)

Theorem 2.1. Let $k$ be an integer greater than two. Suppose that $u \in C^{\infty}\left(\mathbb{S}^{k}, \mathbb{S}^{k}\right)$ is a harmonic map. If $u$ is a conformal diffeomorphism, then $u$ is an isometry. That $i$, there exits $a(k+1) \times(k+1)$ orthogonal matrix $R$ satisfying

$$
u(x)=R x, \quad x \in \mathbb{S}^{k} .
$$


The next lemma is proved in [Xin 1996], but we include the proof here for the convenience of the reader.

Lemma 2.1. Let $u: \mathbb{S}^{k} \rightarrow \mathbb{S}^{k}$ be a harmonic map. For any smooth function $f$ : $\mathbb{S}^{k} \rightarrow \mathbb{R}$, we have

$$
\int_{\mathbb{S}^{k}}|\nabla f|^{2} d \mu-\frac{k-2}{k} \int_{\mathbb{S}^{k}}|d u|^{2}|f(x)|^{2} d \mu \geq \lambda_{1}(u) \int_{\mathbb{S}^{k}}|f|^{2} d \mu
$$

Proof. Let $\left\{\mathbf{e}_{i}\right\}_{i=1}^{k+1}$ be a canonical basis of $\mathbb{R}^{k+1}$. For any $1 \leq i \leq k+1$ we define $\mathbf{e}_{i}^{\top} \in C^{\infty}\left(u^{-1} T \mathbb{S}^{k}\right)$ by

$$
\mathbf{e}_{i}^{\top}(x)=\mathbf{e}_{i}(x)-\left\langle\mathbf{e}_{i}, u(x)\right\rangle u(x)
$$

where $\langle$,$\rangle is a Riemannian metric of \mathbb{R}^{k+1}$. For $f \in C^{\infty}\left(\mathbb{S}^{k}\right)$, we put

$$
\phi_{i}(x)=f(x) \mathbf{e}_{i}^{\top}(x)
$$

By the definition of $\lambda_{1}(u)$, we have

$$
\sum_{i=1}^{k+1} \delta_{u}^{2} \mathbf{E}\left(\phi_{i}\right) \geq \lambda_{1}(u) \sum_{i=1}^{k+1}\left\|\phi_{i}\right\|_{L^{2}\left(\mathbb{S}^{k}\right)}^{2}
$$

Let $\left\{\sigma_{\alpha}\right\}_{\alpha=1}^{k}$ be an orthogonal basis of $T_{x} \mathbb{S}^{k}$. By a direct calculation, we have

$$
\nabla_{\sigma_{\alpha}}^{\prime} \mathbf{e}_{i}^{\top}=u_{i} d u\left(\sigma_{\alpha}\right)
$$

Therefore we have

$$
\nabla_{\sigma_{\alpha}}^{\prime} \phi_{i}=\left(\sigma_{\alpha} f\right) \mathbf{e}_{i}^{\top}+f u_{i} d u\left(\sigma_{\alpha}\right)
$$

Since $\sum_{i=1}^{k+1} \mathbf{e}_{i}^{\top} u_{i}=0$, we have

$$
\sum_{i=1}^{k+1} \sum_{\alpha=1}^{k}\left|\nabla_{\sigma_{\alpha}}^{\prime} \phi_{i}\right|^{2}=\sum_{i=1}^{k+1} \sum_{\alpha=1}^{k}\left\{\left|\left(\sigma_{\alpha} f\right) \mathbf{e}_{i}^{\top}\right|^{2}+\left|f u_{i} d u\left(\sigma_{\alpha}\right)\right|^{2}\right\}=k|\nabla f|^{2}+|d u|^{2}|f|^{2} .
$$

On the other hand

$$
\sum_{i=1}^{k+1}\left|\phi_{i}\right|^{2}=|f|^{2} \sum_{i=1}^{k+1}\left(1-u_{i}^{2}\right)=k|f|^{2}
$$


and we have

$$
\begin{aligned}
\sum_{i=1}^{k+1}\left\langle\operatorname{Tr} R\left(\phi_{i}, d u\right) d u, \phi_{i}\right\rangle & =\sum_{i=1}^{k+1} \sum_{\alpha=1}^{k}\left(\left|d u\left(\sigma_{\alpha}\right)\right|^{2}\left|\phi_{i}\right|^{2}-\left\langle d u\left(\sigma_{\alpha}\right), \phi_{i}\right\rangle^{2}\right) \\
& =k|d u|^{2}|f|^{2}-\sum_{i=1}^{k+1} \sum_{\alpha=1}^{k}\left\langle d u\left(\sigma_{\alpha}\right), f \mathbf{e}_{i}^{\top}\right\rangle^{2} \\
& =(k-1)|d u|^{2}|f|^{2} .
\end{aligned}
$$

Thus the left hand side of (2-1) is

$$
k \int_{\mathbb{S}^{k}}|\nabla f|^{2} d \mu-(k-2) \int_{\mathbb{S}^{k}}|d u|^{2}|f|^{2} d \mu .
$$

The right hand side of (2-1) is

$$
k \lambda_{1}(u) \int_{\mathbb{S}^{k}}|f|^{2} d \mu
$$

and we obtain the desired inequality.

Let $H^{1}\left(\mathbb{S}^{k}\right)$ be the completion of $C^{\infty}\left(\mathbb{S}^{k}\right)$ with respect to the norm

$$
\|f\|_{H^{1}\left(\mathbb{S}^{k}\right)}=\left(\int_{\mathbb{S}^{k}}|f|^{2} d \mu+\int_{\mathbb{S}^{k}}|\nabla f|^{2} d \mu\right)^{1 / 2} .
$$

We define a functional $J: H^{1}\left(\mathbb{S}^{k}\right) \rightarrow \mathbb{R}$ by

$$
J(f)=\int_{\mathbb{S}^{k}}|\nabla f|^{2} d \mu-\frac{k-2}{k} \int_{\mathbb{S}^{k}}|f|^{2} d \mu .
$$

By Lemma 2.1,

$$
J(f) \geq \lambda_{1}(u)\|f\|_{L^{2}\left(\mathbb{S}^{k}\right)}^{2} \quad \text { for any } f \in H^{1}\left(\mathbb{S}^{k}\right) .
$$

The next lemma was proved in [Lin and Wang 2006] and [Okayasu 1994]. See also [Nakajima 1989].

Lemma 2.2. Let $u: \mathbb{S}^{k} \rightarrow \mathbb{S}^{k}$ be a harmonic map.

$$
|\nabla d u|^{2} \geq \frac{k}{k-1}|\nabla| d u||^{2}
$$

Finally, we recall the Bochner formula of harmonic maps between spheres. For the proof, see [Eells and Lemaire 1983; Urakawa 1993; Xin 1996]. 
Lemma 2.3. Let $u: \mathbb{S}^{k} \rightarrow \mathbb{S}^{k}$ be a harmonic map. Then $\frac{1}{2} \Delta\left(|d u|^{2}\right)$

$$
=|\nabla d u|^{2}+(k-1)|d u|^{2}-\sum_{\alpha, \beta=1}^{k}\left(\left|d u\left(\sigma_{\alpha}\right)\right|^{2}\left|d u\left(\sigma_{\beta}\right)\right|^{2}-\left\langle d u\left(\sigma_{\alpha}\right), d u\left(\sigma_{\beta}\right)\right\rangle^{2}\right),
$$

where $\left\{\sigma_{\alpha}\right\}_{\alpha=1}^{k}$ is an orthonormal local frame on $\mathbb{S}^{k}$.

Proof of Theorem 1.1. We use the method in [Nakajima 2006]. Since $\lambda_{1}(u)=$ $2-k<0, u$ is not a constant map. Therefore $e(u)$ is not identically zero. For small $\epsilon>0$, we define $e_{\epsilon}(u)$ by

$$
e_{\epsilon}(u)=e(u)+\epsilon,
$$

and set

$$
\theta=\frac{k-2}{k-1}>0
$$

By direct calculation, we have

$$
\Delta\left(e_{\epsilon}(u)^{\theta}\right)=\theta(\theta-1) e_{\epsilon}(u)^{\theta-2}\left|\nabla e_{\epsilon}(u)\right|^{2}+\theta e_{\epsilon}(u)^{\theta-1} \Delta e_{\epsilon}(u) .
$$

From Lemma 2.2 and Lemma 2.3 we have

$$
\begin{aligned}
\frac{1}{2} \Delta e(u) & =|\nabla d u|^{2}+(k-1)|d u|^{2}-|d u|^{4}+\sum_{\alpha, \beta=1}^{k}\left\langle d u\left(\sigma_{\alpha}\right), d u\left(\sigma_{\beta}\right)\right\rangle^{2} \\
& \geq\left.\frac{k}{k-1}|\nabla| d u\right|^{2}+(k-1)|d u|^{2}-|d u|^{4}+\sum_{\alpha=1}^{k}\left|d u\left(\sigma_{\alpha}\right)\right|^{4} \\
& \geq\left.\frac{k}{k-1}|\nabla| d u\right|^{2}+(k-1)|d u|^{2}-\frac{k-1}{k}|d u|^{4} .
\end{aligned}
$$

Here we used the elementary inequality

$$
\sum_{\alpha, \beta=1}^{k}\left\langle d u\left(\sigma_{\alpha}\right), d u\left(\sigma_{\beta}\right)\right\rangle^{2} \geq \sum_{\alpha=1}^{k}\left|d u\left(\sigma_{\alpha}\right)\right|^{4} \geq \frac{1}{k}\left(\sum_{\alpha=1}^{k}\left|d u\left(\sigma_{\alpha}\right)\right|^{2}\right)^{2}
$$

Combining (2-2) and (2-3), we have

$$
\begin{aligned}
\Delta\left(e_{\epsilon}(u)^{\theta}\right) \geq- & \left.\frac{4(k-2)}{(k-1)^{2}} e_{\epsilon}(u)^{\theta-2}|d u|^{2}|\nabla| d u\right|^{2} \\
& +\frac{2(k-2)}{k-1} e_{\epsilon}(u)^{\theta-1}\left(\left.\frac{k}{k-1}|\nabla| d u\right|^{2}+(k-1)|d u|^{2}-\frac{k-1}{k}|d u|^{4}\right) \\
\geq & \left.2 \theta^{2} e_{\epsilon}(u)^{\theta-2}|d u|^{2}|\nabla| d u\right|^{2}+2(k-2) e(u)^{\theta}-\frac{2(k-2)}{k} e_{\epsilon}(u)^{\theta}|d u|^{2} \\
= & 2\left(\left|\nabla\left(e_{\epsilon}(u)^{\theta / 2}\right)\right|^{2}+(k-2) e(u)^{\theta}-\frac{k-2}{k}|d u|^{2} e_{\epsilon}(u)^{\theta}\right) .
\end{aligned}
$$


Integrating this on $\mathbb{S}^{k}$, we obtain

$$
\int_{\mathbb{S}^{k}}\left|\nabla\left(e_{\epsilon}(u)^{\theta / 2}\right)\right|^{2} d \mu-\frac{k-2}{k} \int_{\mathbb{S}^{k}}|d u|^{2} e_{\epsilon}(u)^{\theta} d \mu \leq(2-k) \int_{\mathbb{S}^{k}} e(u)^{\theta} d \mu .
$$

In particular,

$$
\int_{\mathbb{S}^{k}}\left|\nabla\left(e_{\epsilon}(u)^{\theta / 2}\right)\right|^{2} d \mu \leq \frac{k-2}{k} \int_{\mathbb{S}^{k}}|d u|^{2} e_{\epsilon}(u)^{\theta} d \mu .
$$

From this inequality, we have

$$
\int_{\mathbb{S}^{k}}\left|\nabla\left(e_{\epsilon}(u)^{\theta / 2}\right)\right|^{2} d \mu \leq C,
$$

where $C>0$ is a constant independent of small $\epsilon>0$. Since $e_{\epsilon}(u)$ converges to $e(u)$ at every point of $\mathbb{S}^{k}, e(u)$ belongs to $H^{1}\left(\mathbb{S}^{k}\right)$. By Fatou's lemma, we have

$$
\int_{\mathbb{S}^{k}}\left|\nabla\left(e(u)^{\theta / 2}\right)\right|^{2} d \mu \leq \liminf _{\epsilon \searrow 0} \int_{\mathbb{S}^{k}}\left|\nabla\left(e_{\epsilon}(u)^{\theta / 2}\right)\right|^{2} d \mu .
$$

Thus

$$
\int_{\mathbb{S}^{k}}\left|\nabla\left(e(u)^{\theta / 2}\right)\right|^{2} d \mu-\frac{k-2}{k} \int_{\mathbb{S}^{k}}|d u|^{2} e(u)^{\theta} d \mu \leq(2-k) \int_{\mathbb{S}^{k}} e(u)^{\theta} d \mu .
$$

Since $\lambda_{1}(u)=2-k$, this inequality must be an equality. Thus $e(u)^{\theta / 2}$ is a minimizer of the functional $J$. This implies that $e(u)^{\theta / 2}$ is a solution of the Euler-Lagrange equation of $J$, that is,

$$
-\Delta e(u)^{\theta / 2}-\frac{k-2}{k}|d u|^{2} e(u)^{\theta / 2}=(2-k) e(u)^{\theta / 2} \text { in } \mathbb{S}^{k} .
$$

If $e(u)^{\theta / 2}$ has a zero point $p \in \mathbb{S}^{k}$, then for small $r>0$, we have

$$
\sup _{\mathbb{B}_{r}(p)} e(u)^{\theta / 2} \leq C \inf _{\mathbb{B}_{r}(p)} e(u)^{\theta / 2}=0
$$

by the Harnack inequality, where $\mathbb{B}_{r}(p)$ is an open geodesic ball in $\mathbb{S}^{k}$ of radius $r$ centered at $p$, and $C>0$ is a constant. Thus $u$ is constant in $\mathbb{B}_{r}(p)$ and this implies that $u$ is a constant map by a unique continuation theorem for harmonic maps [Sampson 1978]. This contradicts the fact that $u$ is not a constant map, therefore $e(u)^{\theta / 2}$ has no zero points. On the other hand, considering (2-4), we have

$$
\begin{array}{ll}
\left|d u\left(\sigma_{\alpha}\right)\right|=\left|d u\left(\sigma_{\beta}\right)\right| & (1 \leq \alpha, \beta \leq k), \\
\left\langle d u\left(\sigma_{\alpha}\right), d u\left(\sigma_{\beta}\right)\right\rangle=0 & (\alpha \neq \beta) .
\end{array}
$$

This implies that $d u(x)$ is a conformal isomorphism between $T_{x} \mathbb{S}^{k}$ and $T_{u(x)} \mathbb{S}^{k}$ for any $x \in \mathbb{S}^{k}$, so $u$ is a covering map by the inverse function theorem. Since $\mathbb{S}^{k}$ is 
simply connected, $u$ is a diffeomorphism of $\mathbb{S}^{k}$. Therefore $u$ must be a conformal diffeomorphism of $\mathbb{S}^{k}$.

We complete the proof using Theorem 2.1.

\section{Acknowledgment}

The author thanks the referee for helpful suggestions.

\section{References}

[Eells and Lemaire 1983] J. Eells and L. Lemaire, Selected topics in harmonic maps, CBMS Regional Conference Series in Mathematics 50, Amer. Math. Soc., Providence, 1983. MR 85g:58030 Zbl 0515.58011

[Lin and Wang 2006] F. H. Lin and C. Y. Wang, "Stable stationary harmonic maps to spheres", Acta Math. Sin. (Engl. Ser.) 22:2 (2006), 319-330. MR 2006m:58022 Zbl 1121.58017

[Nakajima 1989] H. Nakajima, "Yau's trick", Sūgaku 41:3 (1989), 253-258. MR 91j:58173 Zbl 0736.53041

[Nakajima 2003] T. Nakajima, Stability and singularities of harmonic maps into spheres, Tohoku Math. Publications 26, Tohoku University Mathematical Institute, Sendai, 2003. MR 2004f:58020 Zbl 1036.53043

[Nakajima 2006] T. Nakajima, "Singular points of harmonic maps from 4-dimensional domains into 3-spheres”, Duke Math. J. 132:3 (2006), 531-543. MR 2007b:58027 Zbl 1097.53044

[Okayasu 1994] T. Okayasu, "Regularity of minimizing harmonic maps into $S^{4}, S^{5}$ and symmetric spaces”, Math. Ann. 298:2 (1994), 193-205. MR 94k:58036 Zbl 0788.58017

[Ramanathan 1986] J. Ramanathan, "A remark on the energy of harmonic maps between spheres", Rocky Mountain J. Math. 16:4 (1986), 783-790. MR 88e:58025 Zbl 0611.58022

[Sampson 1978] J. H. Sampson, "Some properties and applications of harmonic mappings", Ann. Sci. École Norm. Sup. (4) 11:2 (1978), 211-228. MR 80b:58031 Zbl 0392.31009

[Urakawa 1993] H. Urakawa, Calculus of variations and harmonic maps, Translations of Mathematical Monographs 132, American Mathematical Society, Providence, RI, 1993. MR 95c:58050 Zbl 0799.58001

[Xin 1980] Y. L. Xin, "Some results on stable harmonic maps", Duke Math. J. 47:3 (1980), 609-613. MR 81j:58041 Zbl 0513.58019

[Xin 1996] Y. Xin, Geometry of harmonic maps, Progress in Nonlinear Differential Equations and their Applications, 23, Birkhäuser, Boston, 1996. MR 97h:58052 Zbl 0848.58014

Received May 8, 2008. Revised October 29, 2008.

TÔRU NAKAJIMA

DEPARTMENT OF APPLIED MATHEMATICS

FACULTY OF ENGINEERING

SHIZUOKA UNIVERSITY

HAMAMATSU

SHIZUOKA 432-8561

JAPAN

ttnakaj@ipc.shizuoka.ac.jp 